\title{
Prediction of hearing recovery in sudden deafness treated with intratympanic steroids
}

\author{
Previsione delle possibilità di recupero uditivo nell'ipoacusia improvvisa \\ trattata con steroidi intratimpanici
}

\author{
G. ATTANASIO ${ }^{1}$, F.Y. RUSSO ${ }^{2,3}$, E. DI PORTO ${ }^{4}$, L. CAGNONI², E. MASCl ${ }^{2}$, M. RALLI ${ }^{5}$, A. GRECO², \\ M. DE VINCENTIIS 2 \\ ${ }^{1}$ Head and Neck Department, Umberto I Policlinic of Rome, Italy; ${ }^{2}$ Department of Sensory Organs, Sapienza \\ University of Rome, Italy; ${ }^{3}$ AP-HP, Pitié-Salpêtrière Hospital, Department of Otology Auditory Implants and Skull \\ Base Surgery, Paris, France; ${ }^{4}$ Department of Economics and Statistics, Federico II University of Naples, Italy; \\ ${ }^{5}$ Department of Oral and Maxillofacial Sciences, Sapienza University of Rome, Italy
}

\section{SUMMARY}

The present study aims to obtain a probability model allowing prediction of auditory recovery in patients affected by sudden sensorineural hearing loss treated exclusively with intratympanic steroids. A monocentric retrospective chart review of 381 patients has been performed. A Probit model was used to investigate the correlation between the success of treatment (marked or total recovery according to Furuashi's criteria) and the delay between onset of disease and beginning of therapy. The age of patients and audiometric curve shapes were included in the analysis. The results show that delay is negatively correlated with variable success. Considering the entire sample, each day of delay decreases the probability of success by $3 \%$. The prediction model shows that for each day that passes from the onset of the disease the probability of success declines in absence of the medical treatment, hence we conclude that early treatment is strongly recommended.

KEY WORDS: Hearing loss $\bullet$ Sensorineural $\bullet$ Prognosis $\bullet$ Recovery $\bullet$ Audiometry $\bullet$ Steroids

\section{RIASSUNTO}

Il presente studio ha lo scopo di ottenere un modello di probabilità che consenta di prevedere il recupero uditivo nei pazienti affetti da ipoacusia improvvisa neurosensoriale trattati esclusivamente con steroidi intratimpanici. È stata realizzata una revisione retrospettica dei dati di 381 pazienti. Un modello Probit è stato utilizzato per studiare la correlazione tra il successo del trattamento ("marked" or "total recovery" secondo i criteri di Furuashi) e il ritardo (Delay) tra l'insorgenza della malattia e l'inizio della terapia. Sono stati inclusi nell'analisi i dati relativi all'età dei pazienti e alle curve audiometriche. Dallo studio è emerso che la variabile Delay è negativamente correlata con la variabile successo. Considerando l'intero campione, ogni giorno di ritardo diminuisce del 3\% la probabilità di successo. Il modello di previsione mostra che per ogni giorno che passa dal momento della comparsa della malattia, la probabilità di successo declina in assenza dell'intervento; possiamo quindi concludere che il trattamento precoce è fortemente raccomandato.

PAROLE CHIAVE: Ipoacusia $\bullet$ Neurosensoriale $\bullet$ Prognosi $\bullet$ Recupero $\bullet$ Audiometria $\bullet$ Steroidi

Cite this article as: Acta Otorhinolaryngol Ital 2018;38:453-459. http://dx.doi.org/10.14639/0392-100X-1614

(C) Società Italiana di Otorinolaringologia e Chirurgia Cervico-Facciale

\section{Introduction}

Idiopathic sudden sensorineural hearing loss (SSNHL) is defined as a decrease of hearing affecting 3 or more frequencies by $30 \mathrm{~dB}$ or greater over 72 hours or less with no identifiable aetiology.

During 2006 and 2007, the annual incidence of SSNHL was 27 per 100,000 in the United States. The incidence increased with age, ranging from 11 per 100,000 for patients younger than 18 years to 77 per 100,000 for patients aged 65 years and older. There was an overall slight male preponderance with a male-to-female ratio of 1.07:1. This was more pronounced in patients aged 65 years and older, with a ratio of $1.30: 1^{1}$.

The aetiology and natural history of SSNHL are still obscure; many studies investigated the percentage of spontaneous recovery that ranges between 30 and $65 \%$ of cases ${ }^{2}$. However, the real number of patients that recover spontaneously from SSNHL is currently unknown, since many who recover spontaneously within the first days do not 
seek medical treatment. Therefore, the boundary between spontaneous recovery and the efficacy of early medical therapy is still controversial, making the treatment of this condition a current matter of debate ${ }^{34}$.

Various therapies have been proposed without a universally accepted standard protocol. Corticosteroids, antiviral agents, vasodilators, hyperbaric oxygen therapy (HBOT), anticoagulants, anti-inflammatory drugs and other approaches have been suggested, alone or combined, with variable percentages of efficacy reported in literature ${ }^{5-9}$

Despite the controversies in the medical management of SSNHL ${ }^{10}{ }^{11}$, systemic steroid therapy is currently the most widely accepted treatment ${ }^{12-14}$; however, the high dose required for systemic treatment can lead to early and late complications.

Intratympanic (IT) steroid therapy can potentially provide organ-specific treatment with application of high doses of drug directly in the middle ear over the round window membrane, thereby avoiding the adverse effects of systemic corticosteroid therapy ${ }^{15-18}$. The efficacy of IT steroid therapy has already been demonstrated in prospective, randomised, placebo-controlled trials ${ }^{15}{ }^{19}$. Moreover, the American Academy of Otolaryngology-Head and Neck Surgery (AAO-HNS) guidelines for SSNHL recommend that clinicians should offer IT steroid perfusion when patients have incomplete recovery from SSNHL after failure of initial management ${ }^{14}$.

The existing scientific literature sets the timeframe within which maximum recovery may occur from few days to several months, complicating the evaluation of the efficacy of treatment compared to the natural history of the disease. Early steroid treatment has been shown to give greater chances of recovery, especially if performed within the first two weeks from onset; however, timely treatment often does not happen, as delay in diagnosis is a common issue in SSNHL. Moreover, SSNHL can present in different audiometric curves, each having a different course of disease and response to therapy ${ }^{18}$.

To date, the influence of the time to initiation of treatment on hearing prognosis has not been clearly established. The aim of this retrospective study was to propose a probability model that allows predicting the course of auditory recovery in patients suffering from SSNHL treated with IT steroid therapy as first-line treatment. To define the model, we have analysed the course of SSNHL in all patients treated in the last 5 years in a tertiary referral centre, dividing them according to the timing of initiation of therapy, and considering 4 different audiometric curves of presentation.

\section{Materials and methods}

The medical charts of 401 patients diagnosed with SSNHL in the Otolaryngology Department of our Hospital from January 2009 to January 2014 were retrospectively reviewed. Since the present was a retrospective observational study, the institutional ethics committee did not require formal approval. All patients were affected by unilateral SSNHL and treated on an outpatient basis with IT steroid therapy as first-line therapy. The following data were extracted: patient demographics; delay between onset of symptoms and beginning of therapy; audiometric data. We excluded patients lost to follow-up and patients with a subsequent diagnosis of Meniere's disease or cerebellopontine angle tumour (MRI was performed in all patients to rule out a retrocochlear pathology). Overall, 381 patients were included in the study. All patients provided informed consent for the use of clinical data.

\section{Audiometric data}

Patients were evaluated using standardised methods for pure tone threshold audiometry. Pure tone average (PTA) was calculated as the mean of thresholds at 6 frequencies $(250 ; 500 ; 1,000 ; 2,000 ; 4,000$ and $8,000 \mathrm{~Hz})$. Thresholds that could not be measured due to the limit of the audiometric equipment were "dummy coded" with the highest test level of audiometric equipment (5 patients), as suggested in previous studies ${ }^{12}{ }^{19}$. In the present series, this limit was set at $130 \mathrm{~dB}$ HL. Based on the PTA of the pre-treatment audiometric test, patients were distributed into four audiometric curve groups: up-sloping (low frequencies affected), down-sloping (high frequencies affected), flat moderate to severe (all frequencies involved with PTA between 40 and $90 \mathrm{~dB}$ ) and profound (flat audiogram with PTA more than $90 \mathrm{~dB}$ ).

The evaluation of hearing improvement was performed using Furuhashi criteria ${ }^{20}$ (Table I). The outcomes were classified as successful treatment (complete recovery or marked improvement), slight improvement, or no recovery.

Table I. Furuhashi's criteria used to define audiological improvement ${ }^{20}$

\begin{tabular}{ll}
\hline Complete recovery * & PTA ${ }^{* *} \leq 25 \mathrm{~dB}$ or identical to the \\
& contralateral, non-affected ear \\
Marked improvement * & PTA improvement $>30 \mathrm{~dB}$ \\
Slight improvement & PTA improvement between 10 and $30 \mathrm{~dB}$ \\
No recovery & PTA improvement $<10 \mathrm{~dB}$ \\
\hline
\end{tabular}

${ }^{*}$ Complete recovery or marked improvement $=$ successful treatment; ${ }^{* *}$ PTA $=$ sixfrequency pure-tone average (250; 500; 1,000; 2,000; 4,000; 8,000 Hz). 


\section{Therapeutic protocol}

The therapeutic protocol consisted in the IT administration of Prednisolone (Deltacortene Sol ${ }^{\circledR}$, Bruno Farmaceutici, Rome, Italy) at a dose of $0.4 \mathrm{ml}$ of $62.5 \mathrm{mg}$ / $\mathrm{ml}$, once a day for 3 consecutive days. The IT injection procedure, already described in a previous study ${ }^{6}$, was performed on an outpatient basis. The integrity of the tympanic membrane was evaluated with the aid of a microscope. Local anaesthesia was performed by means of a cotton-sponge soaked with $10 \%$ lidocaine solution (Xylocaine, $10 \mathrm{mg} / \mathrm{dose}$, AstraZeneca Korea, Seoul, Korea) placed on the tympanic membrane. The sponge was removed after 20 minutes and the external canal was cleared of any remaining fluid. Patients were placed in a supine position, with their head tilted $40-45^{\circ}$ to the healthy side and then a 25-gauge spinal needle was introduced in the posterior-inferior quadrant of the tympanic membrane. The steroid saline solution was gently perfused into the middle ear. Following the injection, patients were asked to avoid moving their head, speaking or swallowing for 30 minutes.

\section{Statistical analysis}

Our dataset is a cross section of 381 observations, each one reporting information for a single patient.

The main objective was to investigate the relationship between the success variable and the interval between the onset of the sudden deafness and the beginning of treatment (named delay). The data includes only treated patients but there is heterogeneity in their behavior, and therefore we could estimate the correlation between delay and success.

Given the nature of success, which is a dichotomous variable expressing the event of a therapeutic success, it seems obvious to specify a non-linear model. Hence, we used a Probit model for our cross section of individual.

Our baseline specification was

$$
\operatorname{Pr}(\mathrm{Y}(\mathrm{i})=1 \mid \mathrm{X}(\mathrm{i}))=\Phi(\mathrm{X}(\mathrm{i}) * \beta)\{1\}
$$

where Pr represents probability, and $\Phi$ is the Cumulative Distribution Function of the standard normal distribution. The parameters $\beta$ are estimated by maximum likelihood. $\mathrm{X}$ is the matrix of covariates that include age, delay and female.

Another variable has been added to the $\mathrm{X}$ matrix, which may help to understand the role of delay as a determinant of the success rate. We called this variable delay-square, which is computed as the square of delay.

We have defined such a covariate because we believe that the effect of delaying one day can be very heterogeneous over time. Precisely, we believe that a one-day delay at the second day from the onset of SSNHL cannot be compared, in terms of predicted probability of success, to a one-day delay occurring after 30 days from onset. What we expect is that the probability of success decreases quickly during the first days and then decreases more slowly as time passes. Thus, the variable delay will capture the negative linear trend of probability over time (i.e. delaying one day is expected to be always better than delaying one week) and delay-square will capture a difference in the slope of the predicted probability over time (i.e. a one day delay at the first day from the onset will decrease the probability of success by more than a one-day delay after a week from the onset). We expect to find a negative and significant sign for delay, while we expect a positive and significant sign for delay-square. Moreover, such specifications allow better determination of when the therapy will likely be unsuccessful, and therefore of how many days of delay are tolerable to provide a successful treatment.

Model $\{1\}$ has been applied to several sub-samples. We have firstly developed a general model including all the observations in the sample; afterwards, we divided the sample according to the variable pattern. This is a categorical variable with 4 values: up-sloping, down-sloping, flat moderate to severe and profound.

\section{Results}

Three-hundred eighty-one patients were included in the present study. The mean age was $50.7 \pm 16$ years (range: 11-92 years). The sample included 168 females (44.1\%) and 213 males (65.9\%), the mean delay was 17.5 days \pm 19 . A standard deviation of 19.5 days for this variable shows very heterogeneous patient behaviour. The variable delay has a median of 9 days and a maximum of 100 ; a large part of our sample presented during the first week after SSNHL onset.

Overall, the therapy had a success rate of $47.2 \%$; however, a standard deviation of 0.49 for this variable suggests that the probability of having a positive outcome is a complex event that should be related to specific individual characteristics.

Table II shows the summary of statistics for the main variables used for the analysis: age of the patient; delay, indicating the number of days between the onset of SSNHL and the beginning of IT therapy; two dummy variables, female $=1$ when the patient is female gender, and success $=1$ when the outcome of the Furuhashi index collected at the end of the therapy showed a complete or marked recovery.

Considering the 4 different audiometric curves, we supposed that the probability of success may be heterogeneous within the different patterns, and thus we can use the different values of success rate to identify different sub- 
Table II. Main variables analysed in the present study.

\begin{tabular}{lcccc} 
& Age & Female & Delay & Success \\
Mean & 50.72 & $44.6 \%$ & 17.56 & $47.26 \%$ \\
p50 & 50 & 0 & 9 & 0 \\
Min & 11 & 0 & 1 & 0 \\
Max & 92 & 1 & 100 & 1 \\
SD & 16.212 & 0.497 & 19.502 & 0.499 \\
\hline
\end{tabular}

p 50: median; min: minimum value; max: maximum value; SD: standard deviation; age: age of the patient; delay: number of days between the onset of SSNHL and the beginning of IT therapy; two dummy variables, female $=1$ when the patient is female gender, and success $=1$ when the outcome of the Furuhashi index collected at the end of the therapy shows a complete or marked recovery.

populations. We also expect that the delay coefficients will be very different over the different patterns. Thus, the treatment will heterogeneously affect the different subpopulations.

Table III shows the marginal effects from the Probit regressions for the equation $\{1\}$. Marginal effect is a way of estimating how much the event probability changes when a given predictor is changed by one unit. In our study, the marginal effect of the covariates is defined as the change in success rate when a covariate changes by one unit. Column 1 includes the data concerning the whole sample, and columns 2 to 5 show results for the different sub-samples; precisely the sub-samples are tailored on different patterns (i.e. flat moderate to severe, up-sloping, down-sloping, profound). The results show that delay and delay-square are highly significant in columns 1 to 3 , and delay is significant in columns 1 to 4 . These results can be deduced by the number of observations in each sample. Therefore, it is expected that the first column shows strongly significant results.

In our sample, females showed a greater tendency to recover compared to men. This difference is around $10 \%$ on average in the entire sample; in the profound pattern group, females showed $30 \%$ more probability of success than men. Age appeared to be always negatively correlated with the probability of success.

The predicted probability of success generated by the specified model, slightly different among the different samples, is between 0.40 and 0.54 . In particular, the upsloping pattern (0.54) appeared to be the one with higher chances of recovery. On the base of the aforementioned results, a probability model was realised to exploit the statistical model; Table IV shows the chances of success predicted by this model considering five age classes for different delay values. For instance, patients aged between 31 and 50 years have a probability of recovery of $77 \%$ if the treatment is initiated after one day from the onset of SSNHL, this probability decreases to $50 \%$ if the treatment begins after two weeks from SSNHL onset.

\section{Discussion}

SSNHL treatment is a matter of evolving debate. While the current standard of care is widely recognised to be corticosteroid therapy ${ }^{121321}$, the course of disease has not yet been defined with certainty. Recently, a meta-analysis was carried out to investigate the comparison between IT and systemic steroids. Han et al. compared the efficacy of combination therapy (combined IT and systemic use of steroids) with systemic steroid therapy as primary treatment for SSNHL ${ }^{22}$. A total of 14 randomised controlled trials were selected, including 756 subjects allocated to combined therapy and 638 to systemic steroids. The analysis revealed that combined therapy seems to confer a certain degree of benefit as primary treatment of SSNHL compared with only systemic treatment. In a randomised, triple-blind, controlled trial in $2017{ }^{23}$ on 112 patients, 32 received IT corticosteroids, 45 received systemic corticosteroids and 35 received a combination of the two. No differences were found in hearing recovery between the three therapeutic approaches. These results are in line

Table III. Marginal effects from the Probit regressions for the equation $\{1\}$.

\begin{tabular}{|c|c|c|c|c|c|}
\hline Covariate & $\begin{array}{c}\text { (1) ME } \\
\text { Whole sample }\end{array}$ & $\begin{array}{c}\text { (2) ME } \\
\text { Flat moderate to severe }\end{array}$ & $\begin{array}{l}\text { (3) ME } \\
\text { Up-sloping }\end{array}$ & $\begin{array}{c}\text { (4) ME } \\
\text { Down-sloping }\end{array}$ & $\begin{array}{l}\text { (5) ME } \\
\text { Profound }\end{array}$ \\
\hline Delay & $\begin{array}{c}-0.028^{\star \star \star *} \\
(0.0489)\end{array}$ & $\begin{array}{c}-0.044^{\star \star \star} \\
(0.0087)\end{array}$ & $\begin{array}{c}-0.056 \text { ** } \\
(0.0281)\end{array}$ & $\begin{array}{c}-0.0280 \text { ** } \\
(0.0131)\end{array}$ & $\begin{array}{c}-0.0133 \\
(0.0197)\end{array}$ \\
\hline Delaysq & $\begin{array}{c}+0.0001^{\star \star \star} \\
(0.0000)\end{array}$ & $\begin{array}{c}+0.0003^{* \star \star} \\
(0.0001)\end{array}$ & $\begin{array}{c}+0.0007 \text { * } \\
(0.0004)\end{array}$ & $\begin{array}{c}+0.0001 \\
(0.0002)\end{array}$ & $\begin{array}{c}+0.0000 \\
(0.0004)\end{array}$ \\
\hline Female & $\begin{array}{c}+0.1024 \text { * } \\
(0.0604)\end{array}$ & $\begin{array}{c}+0.1333 \\
(0.1006)\end{array}$ & $\begin{array}{c}+0.1336 \\
(0.2199)\end{array}$ & $\begin{array}{c}+0.0157 \\
(0.1148)\end{array}$ & $\begin{array}{c}+0.2869 \text { *** } \\
(0.1325)\end{array}$ \\
\hline Age & $\begin{array}{c}-0.0058 \text { *** } \\
(0.0183)\end{array}$ & $\begin{array}{c}-0.0043 \\
(0.0031)\end{array}$ & $\begin{array}{c}-0.0259 * \star \star \\
(0.0107)\end{array}$ & $\begin{array}{c}-0.0066 \text { * } \\
(0.0036)\end{array}$ & $\begin{array}{r}-0.0017 \\
(0.0036)\end{array}$ \\
\hline Predicted probability of success & 0.45 & 0.40 & 0.54 & 0.40 & 0.43 \\
\hline
\end{tabular}

ME: marginal effect; Standard error in parentheses; ${ }^{\star * \star} p<0.01 ;{ }^{* \star} p<0.05 ;{ }^{*} p<0.1$. 
Table IV. Probability of success predicted by the model considering five age classes and four audiometric curves, at different delay endpoints.

\begin{tabular}{lcccccccccccccccc}
\multicolumn{1}{c}{} & DAY 1 & DAY 2 & Day 3 to 5 & Day 6 to 10 & & & & & & & & & \\
Age (years) & U & D & F & P & U & D & F & P & U & D & F & P & U & D & F & P \\
$16-30$ & 1 & 0.85 & 0.94 & 0.7 & 1 & 0.84 & 0.9 & 0.49 & 0.99 & 0.79 & 0.86 & 0.52 & 0.97 & 0.69 & 0.72 & 0.44 \\
$31-50$ & 0.97 & 0.76 & 0.87 & 0.55 & 0.97 & 0.76 & 0.87 & 0.51 & 0.93 & 0.71 & 0.81 & 0.48 & 0.84 & 0.61 & 0.69 & 0.46 \\
$51-65$ & 0.88 & 0.71 & 0.87 & 0.6 & 0.68 & 0.64 & 0.78 & 0.47 & 0.70 & 0.62 & 0.76 & 0.46 & 0.56 & 0.52 & 0.64 & 0.46 \\
Over 65 & 0.47 & 0.58 & 0.79 & 0.60 & 0.47 & 0.57 & 0.79 & 0.60 & 0.38 & 0.51 & 0.72 & 0.58 & 0.21 & 0.41 & 0.59 & 0.57 \\
AVG & 0.83 & 0.73 & 0.87 & 0.61 & 0.78 & 0.70 & 0.84 & 0.52 & 0.75 & 0.66 & 0.79 & 0.51 & 0.65 & 0.56 & 0.66 & 0.48 \\
\hline
\end{tabular}

U: up-sloping audiometric curve; D: down-sloping audiometric curve; F: flat audiometric curve; P: profound audiometric curve; AVG: probability of success of treatment, the variable success ranges from 0 to 1.

with previous studies performed by our group on 265 patients in $2014{ }^{18}$ and by Rauch et al. on 250 patients in $2011^{24}$. In both studies, patients were treated either by IT or oral steroids, and both treatments showed the same efficacy on SSNHL.

Though SSNHL is commonly considered to be an otologic emergency, there are very few trials that report evidences regarding the urgency of medical care ${ }^{325} 26$. Several factors have been reported to have a prognostic impact on SSNHL. Hearing recovery appears to be related to the degree of initial hearing loss ${ }^{27}$, as well as to age and the shape of the audiogram ${ }^{2526}$.

Many studies have analysed vertigo as a possible predictor of hearing recovery in SSNHL, assessing that subjective perception of vertigo is a predictor for worse outcome ${ }^{25} 26$. Also, according to several reports, advanced age, hypertension, diabetes and hyperlipidaemia are poor prognostic factors; however, there is still no general agreement on the real influence of these factors on recovery ${ }^{28-35}$. In recent years, different studies have investigated the prognostic factors in SSNHL treated by systemic steroids. In 2015, Magnano et al. ${ }^{36}$ found that only precocity of treatment and up-sloping hearing loss were positive prognostic factors for hearing recovery. A recent retrospective study conducted on 494 patients treated with either IT or oral steroids showed that age, severity of initial pure tone audiometry, duration from onset to treatment and initial speech discrimination were statistically significant prognostic factors related to hearing improvement in SSNHL, and that IT steroid injection as an initial single treatment is comparable to systemic oral steroid administration ${ }^{37}$.

In the present study, we proposed a prognostic model able to predict auditory recovery with respect to the precocity of medical treatment, also considering the audiometric curve shape. The decision of choosing an audiometric curve shape classification instead of Clark classification ${ }^{38}$ is believed to be coherent with previous studies published from our group ${ }^{17} 18$. The results of the study and application of the statistical equation show that poorer recovery of hearing can be expected when medical treatment is delayed.

Delay and delay-square are highly significant in the majority of samples. This suggests a nonlinear pattern for the variable delay. As we hypothesised, delay is negatively correlated with the variable success; moreover, delaysquare shows a positive correlation in the entire sample. These two data together suggest that the probability of success decreases with time; however, during the first days following the onset of the SSNHL such a decrease is much more evident. Therefore, we can assume that early treatment is strongly recommended. Considering the entire sample, each day of delay decreases the probability of success by $3 \%$. For the flat curves, the percentage is $4.4 \%$, while for the up-sloping pattern it is 5.6\%. For a downsloping pattern, the probability of success decreases with the same values of the entire sample.

The behaviour of the profound pattern is more difficult to predict due to the lack of robustness in our estimates (lower number of patients). Nevertheless, these patients showed a decrease of $1.3 \%$ in the probability of recovery for each day of delayed treatment.

Figure 1 shows a graph derived by the Probit regressions. In this graph, we plotted the predicted probability as computed using different specifications against the variable delay, following the Probit regression $\{1\}$. The curve is evidently much sharper in the first part of the graph, indicating that for each day that passes from the onset of SSNHL and initiation of treatment the probability of success decreases, and that chances of recovery lower to less than 50\% after the first three weeks. The graph is reliable until the $40^{\text {th }}$ day of delay, since the confidence interval is narrow until that point; this is due to the number of observations that is higher during the first period.

The developed predictive model not only has a theoretical value, but clinical application is also possible. Table IV identifies the probability of success for each age class according to the timing in which the patient begins medical treatment with IT corticosteroids. 


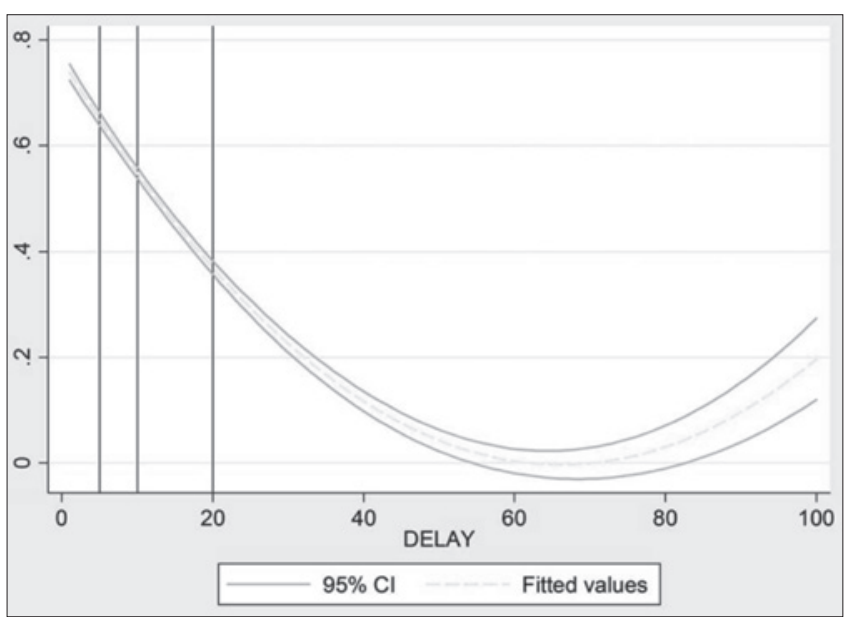

Fig. 1. Effect of delay-square variable on the entire sample.

\section{Conclusions}

The role of precocity of treatment in recovery from SSNHL is still not clear. The statistical analysis performed herein allowed defining a probabilistic model that is able to identify the chances of recovery of patients that begin medical treatment with different timing. The prediction model shows that for each day that passes from the onset of SSNHL to the beginning of treatment, the probability of success decreases, strongly suggesting that early treatment is recommended.

\section{Conflict of interest statement}

None declared.

\section{References}

1 Alexander TH, Harris JP. Incidence of sudden sensorineural hearing loss. Otol Neurotol 2013;34:1586-9.

2 Mattox DE, Simmons FB. Natural history of sudden sensorineural hearing loss. Ann Otol Rhinol Laryngol 1977;86:463-80.

3 Tran Ba Huy P, Sauvaget E. Idiopathic sudden sensorineural hearing loss is not, at this time, an otologic emergency. Ann Otolaryngol Chir Cervicofac 2007;124:66-71.

4 Conlin AE, Parnes LS. Treatment of sudden sensorineural hearing loss. II. A meta-analysis. Arch Otolaryngol Head Neck Surg 2007;133:582-6.

5 Battaglia A, Burchette R, Cueva R. Combination therapy (intratympanic dexamethasone + high-dose prednisone taper) for the treatment of idiopathic sudden sensorineural hearing loss. Otol Neurotol 2008;29:453-60.

6 Filipo R, Covelli E, Balsamo G, et al. Intratympanic prednisolone therapy for sudden sensorineural hearing loss: a new protocol. Acta Otolaryngol 2010;130:1209-13.
7 Tucci DL, Farmer JC Jr, Kitch RD, et al. Treatment of sudden sensorineural hearing loss with systemic steroids and valacyclovir. Otol Neurotol 2002;23:301-8.

8 Filipo R, Attanasio G, Viccaro M, et al. Hyperbaric oxygen therapy with short duration intratympanic steroid therapy for sudden hearing loss. Acta Otolaryngol 2012;132:475-81.

9 Attanasio G, Covelli E, Cagnoni L, et al. Does the addition of a second daily session of hyperbaric oxygen therapy to intratympanic steroid influence the outcomes of sudden hearing loss? Acta Otorhinolaryngol Ital 2015;35:272-6.

10 Crane RA, Camilon M, Nguyen S, et al. Steroids for treatment of sudden sensorineural hearing loss: a meta-analysis of randomized controlled trials. Laryngoscope 2015;125:209-17.

11 Wei BP, Stathopoulos D, O'Leary S. Steroids for idiopathic sudden sensorineural hearing loss. Cochrane Database Syst Rev 2013;7:CD003998.

${ }_{12}$ Chen CY, Halpin C, Rauch SD. Oral steroid treatment of sudden sensorineural hearing loss: a ten year retrospective analysis. Otol Neurotol 2003;24:728-33.

13 Lawrence R, Thevasagayam R. Controversies in the management of sudden sensorineural hearing loss: an evidencebased review. Clin Otolaryngol 2015;40:176-82.

${ }^{14}$ Stachler RJ, Chandrasekhar SS, Archer SM, et al. Clinical practice guideline: sudden hearing loss. Otolaryngol Head Neck Surg 2012;146:S1-35.

15 Banerjee A, Parnes LS. Intratympanic corticosteroids for sudden idiopathic sensorineural hearing loss. Otol Neurotol 2005;26:878-81.

16 Battista RA. Intratympanic dexamethasone for profound idiopathic sudden sensorineural hearing loss. Otolaryngol Head Neck Surg 2005;132:902-5.

17 Filipo R, Attanasio G, Russo FY, et al. Intratympanic steroid therapy in moderate sudden hearing loss: a randomized, triple-blind, placebo-controlled trial. Laryngoscope 2013;123:774-8.

18 Filipo R, Attanasio G, Russo FY, et al. Oral versus shortterm intratympanic prednisolone therapy for idiopathic sudden hearing loss. Audiol Neurootol 2014;19:225-33.

19 Plontke SK, Löwenheim H, Mertens J, et al. Randomized, double blind, placebo controlled trial on the safety and efficacy of continuous intratympanic dexamethasone delivered via a round window catheter for severe to profound sudden idiopathic sensorineural hearing loss after failure of systemic therapy. Laryngoscope 2009;119:359-69.

${ }^{20}$ Furuhashi A, Matsuda K, Asahi K, et al. Sudden deafness: long-term follow-up and recurrence. Clin Otolaryngol Allied Sci 2002;27:458-63.

21 Rauch SD. Clinical practice. Idiopathic sudden sensorineural hearing loss. N Engl J Med 2008;359:833-40.

${ }^{22}$ Han X, Yin X, Du X, et al. Combined intratympanic and systemic use of steroids as a first-line treatment for sudden sensorineural hearing loss: a meta-analysis of randomized, controlled trials. Otol Neurotol 2017;38:487-95.

${ }^{23}$ Ashtiai MK, Firouzi F, Bastaninejad S, et al. Efficacy of sys- 
temic and intratympanic corticosteroid combination therapy versus intratympanic or systemic therapy in patients with idiopathic sudden sensorineural hearing loss: a randomized controlled trial. Eur Arch Otorhinolaryngol 2017.

24 Rauch SD, Halpin CF, Antonelli PJ. Oral vs intratympanic corticosteroid therapy for idiopathic sudden sensorineural hearing loss: a randomized trial. JAMA 2011;305:2071-9.

25 Edizer DT, Çelebi Ö, Hamit B, et al. Recovery of idiopathic sudden sensorineural hearing loss. J Int Adv Otol 2015;11:122-6.

26 Rauch SD, Halpin CF, Antonelli PJ, et al. Oral vs intratympanic corticosteroid therapy for idiopathic sudden sensorineural hearing loss: a randomized trial. JAMA 2011;305:2071-9.

27 Cvorović L, Deric D, Probst R, et al. Prognostic model for predicting hearing recovery in idiopathic sudden sensorineural hearing loss. Otol Neurotol 2008;29:464-9.

28 Park HM, Jung SW, Rhee CK. Vestibular diagnosis as prognostic indicator in sudden hearing loss with vertigo. Acta Otolaryngol Suppl 2001;545:80-3.

29 Harada H, Kato T. Prognosis for sudden sensorineural hearing loss: a retrospective study using logistical regression analysis. Int Tinnitus J 2005;11:115-8.

30 Jarvis SJ, Giangrande V, John G, et al. Management of acute idiopathic sensorineural hearing loss: a survey of UK ENT consultants. Acta Otorhinolaryngol Ital 2011;31:85-9.

31 Ciorba A, Hatzopoulos S, Bianchini C, et al. Idiopathic sud- den sensorineural hearing loss: cardiovascular risk factors do not influence hearing threshold recovery. Acta Otorhinolaryngol Ital 2015;35:103-90.

32 Xie S, Qiang Q, Mei L, et al. Multivariate analysis of prognostic factors for idiopathic sudden sensorineural hearing loss treated with adjuvant hyperbaric oxygen therapy. Eur Arch Otorhinolaryngol 2018;275:47-51.

33 Chen L, Zhang G, Zhang Z, et al. Neutrophil-to-lymphocyte ratio predicts diagnosis and prognosis of idiopathic sudden sensorineural hearing loss: a systematic review and metaanalysis. Medicine (Baltimore) 2018;97:e12492.

34 Russo FY, De Seta D, Lahlou G, et al. Fluctuating hearing loss in the only hearing ear: cochlear implantation in the contralateral deaf side. Otolaryngol Head Neck Surg 2018;158:1101-6.

35 Califano L, Salafia F, Melillo MG, et al. Sensitivity and specificity of vestibular bed-side examination in detecting VIII cranial nerve schwannoma with sensorineural sudden unilateral hearing loss as presenting symptom. Acta Otorhinolaryngol Ital 2017;37:336-40.

36 Magnano M, Orione M, Boffano P, et al. Sudden hearing loss: a study of prognostic factors for hearing recovery. J Craniofac Surg 2015;26:e279-82.

37 Kang WS, Yang CJ, Shim M, et al. Prognostic factors for recovery from sudden sensorineural hearing loss: a retrospective study. J Audiol Otol 2017;21:9-15.

38 Clark JG. Uses and abuses of hearing loss classification. Asha 1981;23:493-500.

Received: January 31, 2017 - Accepted: December 18, 2017

Address for correspondence: Giuseppe Attanasio, Head and Neck Department, ENT Clinic, Umberto I Policlinic, viale del Policlinico 155, 00161 Rome, Italy. E-mail: giuseppe.attanasio@uniroma1.it 